\title{
MODELING NANOCOMPOSITES: FROM RHEOLOGY TO FORMING PROCESSES SIMULATION
}

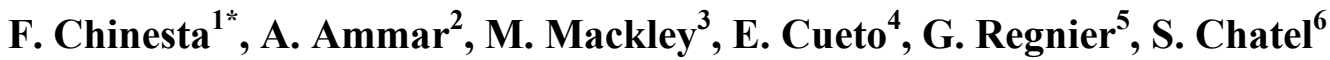 \\ ${ }^{1}$ EADS Corporate International Chair, GeM - CNRS - Ecole Centrale de Nantes, Nantes, France \\ ${ }^{2}$ Université Joseph Fourier, Laboratoire de Rhéologie, UMR CNRS, Grenoble, France \\ ${ }^{3}$ Department of Chemical Engineering and Biotechnology, University of Cambridge, UK \\ ${ }^{4}$ I3A, Universidad de Zaragoza, Spain \\ ${ }^{5}$ LIM - CNRS - ENSAM Paris, France \\ ${ }^{6}$ EADS-IW, Suresnes, France
}

\begin{abstract}
Composites materials reinforced using nano-particles or carbon nano-tubes (CNTs) are more and more used in industry and their modeling is one of the most active research topics in this area. As in usual processes involving composite materials, the main objective is to understand the induced properties (electrical, thermal, mechanical ...) and obviously to optimize process parameters for improving formed parts. Despite the intensive research activity in this field, the modeling of processes involving nano-charges remains a real challenge. In this work we are revisiting the state of the art on this topic, from the rheological modeling of CNTs suspensions to its use in composites forming processes.
\end{abstract}

KEYWORDS: Nanocomposites, Carbon Nanotubes, Rheology, Material Forming, Simulation

\section{INTRODUCTION}

Carbon nanotubes (CNTs) are cylinders of rolled graphite sheets possessing high mechanical strength, low density, and special electronic properties [1]. They belong to a relatively new class of fibrous material that can potentially be used for high-performance nanocomposites and nano-devices. Most of these applications involve suspending the CNTs in a matrix, and thereby allow for further processing such as fibre spinning, film casting, extrusion, and inkjet printing. Both in terms of processing and product, a uniformly dispersed suspension of CNT is generally desirable; however, CNTs have low solubility in most common solvents, and therefore obtaining a homogenous dispersion of CNT can be laborious and difficult. For instance, Huang et al. [2] reported that a mixing time of several hours was required to establish a stable rheology for CNT suspensions and even then, uniformity at nano-scale is not guaranteed. In this regard, introducing functional groups onto the wall/cap of CNTs, also known as functionalisation, has proven to be one of the most effective ways to enhance dispersion of CNT in a processing matrix [3]. Despite the potential of functionalisation, a limited amount of work has been carried out on the rheological modelling of chemically treated CNT suspensions.

In relation to the modelling of forming processes, an understanding in the flow behaviour of CNT suspensions is needed for the development of a suitable constitutive model. The final properties of CNT composites can be very sensitive to the CNT orientation and aggregation states, it would therefore be useful to develop a stressmicrostructure model which contains this information. In terms of applications, a large number of potential applications have been suggested for CNTs, ranging from composites, electronic devices to biomedical applications.

\section{PROCESSES SIMULATION}

Prior to simulate any kind of forming processes involving suspending nanotubes into a matrix fluid, an appropriate flow model should be derived. That model consists of the classical balance equations and an appropriate constitutive law. The momentum equation neglecting mass and inertia terms writes:

$$
\nabla \times \boldsymbol{\sigma}=\mathbf{0}
$$

where $\boldsymbol{\sigma}$ is the Cauchy stress tensor. The mass balance, assuming the suspension incompressible reads:

$$
\nabla \times \mathbf{v}=0
$$

where $\mathbf{V}$ denotes the velocity of the homogenized continuum medium consisting of the matrix and the suspended CNTs. Finally, in order to close the flow

\footnotetext{
* Corresponding author: 1 rue de la Noë, BP 92101, 44321 Nantes - France, (33) 24037 68 84, Francisco.Chinesta@ec-nantes.fr
} 
model a constitutive equation must be introduced whose most general form can be written as:

$$
\boldsymbol{\sigma}=-P \mathbf{I}+2 \eta \mathbf{D}+\boldsymbol{\tau}^{C N T}
$$

where the first two terms on the right hand represent a Newtonian contribution and the last term the contribution to the stress coming from the presence of the CNTs. In that equation $P, \eta$ and $\mathbf{D}$ are the pressure field, the matrix viscosity and the strain rate tensor respectively. Obviously the most delicate point in the establishment of such a constitutive equation is the term due to the CNTs contribution $\boldsymbol{\tau}^{C N T}$. This term must account for the concentration and orientation of the tubes, but also for the dispersion state of CNTs in the suspension.

\section{RHEOLOGY}

\subsection{FUNCTIONALIZED CNTS}

When CNTs are functionalized their aggregation is avoided and then the microstructure state can be described from the tubes orientation that we can describe by introducing the distribution function $\Psi(\mathbf{x}, t, \mathbf{p})$ [4] that represents the fraction of tubes that at position $\mathbf{x}$ and time $t$ are aligned in the direction $\mathbf{p}$ (the unit vector $\mathbf{p}$ is defined on the unit sphere).

The conservation balance of $\Psi(\mathbf{x}, t, \mathbf{p})$ writes:

$$
\frac{\partial \Psi}{\partial t}+\frac{\partial(\dot{\mathbf{x}} \Psi)}{\partial \mathbf{x}}+\frac{\partial(\dot{\mathbf{p}} \Psi)}{\partial \mathbf{p}}=0
$$

where $\dot{\mathbf{x}} \equiv \mathbf{v}$. In rheometric flows $\Psi(t, \mathbf{p})$ and then Eq. (4) reduced to

$$
\frac{\partial \Psi}{\partial t}=-\frac{\partial(\dot{\mathbf{p}} \Psi)}{\partial \mathbf{p}}
$$

In Eq. (5) $\dot{\mathbf{p}}$ represents the rotary tube velocity that is induced by the flow and also from the tube-tube and tube-matrix interactions. These interactions be described from a Brownian term: $\dot{\mathbf{p}}=\dot{\mathbf{p}}^{\text {drift }}+\dot{\mathbf{p}}^{\text {Brow }}$. The first contribution can be modelled from the Jeffery's equation

$$
\dot{\mathbf{p}}^{\text {drift }}=(\nabla \mathbf{v}) \times \mathbf{p}-\left(\mathbf{p}^{T} \times \mathbf{D} \times \mathbf{p}\right) \mathbf{p}
$$

where the tubes were assumed with an infinite aspect ratio. Now, the Brownian contribution can be written as:

$$
\dot{\mathbf{p}}^{\text {Brow }}=-D_{r} \frac{1}{\Psi} \frac{\partial \Psi}{\partial \mathbf{p}}
$$

Now, by introducing Eqs. (6) and (7) into Eq. (5) we obtain the classical Fokker-Planck orientation equation:

$$
\frac{\partial \Psi}{\partial t}=-\frac{\partial\left(\dot{\mathbf{p}}^{\text {drift } \Psi)}\right.}{\partial \mathbf{p}}+D_{r} \frac{\partial^{2} \Psi}{\partial \mathbf{p}^{2}}
$$

that represents an advection-diffusion equation in the conformational coordinate $\mathbf{p}$ (that represents the orientation space).

As soon as the flow kinematics is known from its velocity field $\mathbf{V}$ (from which the strain rate tensor $\mathbf{D}$ can be calculated) Eq. (8) can be integrated and then the tubes orientation distribution computed. However, as Eq. (3) reflects, both, the flows kinematics and the tubes orientation, are coupled from the extra-stress tensor related to the presence of tubes. Thus, an expression relating $\Psi(\mathbf{x}, t, \mathbf{p})$ with $\boldsymbol{\tau}^{C N T}$ is needed. This equation is simply the virial stress (also known as the Kramer's rule):

$$
\boldsymbol{\tau}^{C N T}=\int_{S(0,1)}(\mathbf{F}(\mathbf{p}) \otimes \mathbf{p}) \Psi(\mathbf{p}) d \mathbf{p}
$$

where $S(0,1)$ represents the unit sphere and $\mathbf{F}(\mathbf{p})$ is the force acting on the tubes aligned along $\mathbf{p}$. This force (whose derivation was illustrated in [5]) writes:

$$
\mathbf{F}(\mathbf{p})=\xi\left(\mathbf{p}^{T} \times \mathbf{D} \times \mathbf{p}\right) \mathbf{p}
$$

where $\xi$ is a constant that depends on the friction coefficient, on the concentration and on length of the tubes. In the expression of the extra-stress tensor we neglected the contribution of Brownian effects to the stress, as well as all the possible contributions coming from the tube-tube or tube-matrix interactions due to the tubes functionalization. These effects are not relevant in the case of kinematics implying large deformations, but in the case of small amplitude oscillatory flows those effects must be retained because they are at the origin of the mild elasticity noticed. This topic was deeply discussed in [6].

Now, the flow model is closed and it can be solved. At each time step the velocity field can be updated from the $\boldsymbol{\tau}^{C N T}$ existing at the previous time step. Then, the orientation distribution is updated and the stress is recomputed by using Eq. (9). The incremental strategy continues until reaching the final simulation time or the steady state. This model involves two additional rheological parameters: the constant $\xi$ and the rotary diffusion coefficient $D_{r}$.

\subsection{NON-FUNCTIONALIZED CNTs}

In the case of non-treated CNTs richer microstructures were observed [7][8]. In fact the Van der Waals forces induce tubes aggregation. The aggregates can evolve because by adding news tubes or small aggregates they increase their size. Simultaneously, the strain rate can induce disaggregation. Thus, the microstructure cannot 
be described only from the orientation state; another variable addressing the aggregates distribution is required. In [8] we proposed to enrich the distribution function in order to include both the orientation and the population of aggregates. For this purpose we defined a scalar variable $n \in[0,1]$ that represents the microstructure state ranging from a population composed of free tubes $(n=0)$ to a densely network $(n=1)$. The distribution function writes $\Psi(t, \mathbf{p}, n)$ when the flow is assumed homogeneous (in the general case this distribution function also depends on the physical space coordinates $\mathbf{X}$ ). Now, the conservation balance reads:

$$
\frac{\partial \Psi}{\partial t}+\frac{\partial(\dot{\mathbf{x}} \Psi)}{\partial \mathbf{x}}+\frac{\partial(\dot{\mathbf{p}} \Psi)}{\partial \mathbf{p}}+\frac{\partial(\dot{n} \Psi)}{\partial n}=0
$$

In [7] we proposed a particular form of the last term in Eq. (11), but many other possibilities exist. The Kramer's rule was generalized accordingly and with only two additional rheological parameters an excellent agreement with the experimental measurements was noticed.

\subsection{UNSOLVED CHALLENGES}

Despite the quite good results for explaining the rheology of both treated and untreated CNTs suspensions, numerous challenges remains unsolved. In what follows we summarize the main issues.

\subsubsection{Treated CNTs}

The first challenge concerns the explanation of the noticed mild elasticity in the case of treated CNTs. In [6] we proposed a dependence of the rotary diffusion on the square root of the frequency. This dependence, which was attributed to a kind of network generated by the tubes functionalization, allowed fitting successfully the linear viscoleastic data.

Other authors attributed this behaviour to the tubes bending [9][10]. In general the functionalized CNTs were SWCNT (single wall CNTs) that can be assumed in absence of external forces, quasi perfectly straight. In that case the tubes bending is originated by the random effects and then amplified by the action of the flow that acts with different intensities depending on the orientation of each tube segment. However, at present, bending models have been analyzed in the framework of Brownian dynamics simulations making difficult their use in the complex flows encountered in industrial forming processes. Another way to introduce those effects consists of introducing a Cosserat's framework combined with richer descriptions of the flexible tube.

\subsubsection{Untreated CNTs}

In the case of untreated CNTs the main issue concerns the aggregation/disaggregation kinetics. As we indicated previously a first tentative model was proposed in [8] however numerous better alternatives exist. The other main issue concerns the expression of the rotary velocity induced by the flow that is assumed following the Jeffery equation (the affine deformation also used in most of models involving associative polymers or entangled polymers). In the case of associative polymers it was assumed that molecules belonging to the network orient affinely with the flow whereas they are not subjected to any rotary diffusion effect. On the other hand, free molecules also orient affinely with the flow but they are subjected to rotary diffusion effects. If one considers a similar behaviour for our rods (the CNTs) the more entangled is the population, the higher is the order parameter (the order parameter quantify de degree of alignment of tubes in a certain direction, that in simple shear flows corresponds to the flow direction). However, different authors noticed the opposite tendency. This behaviour could be explained by assuming that the more entangled are the tubes, the higher is the delay for aligning in the flow direction. Thus, until now, we considered a rotary diffusion increasing with $n$ : the more the tubes are entangled, the higher is the isotropy of the orientation distribution induced by the rotary diffusivity. Another possibility lies in introducing a delay in the Jeffery's equation. However this alternative is quite delicate. Different attempts have been proposed for introducing such a delay by assuming that the velocity that appears in the Jeffery's equation is only a fraction of the real one. However the resulting models violate the objectivity principle. There are other attempts for introducing the delay without violating the objectivity principle, but in our knowledge no model considers an expression of the stress in agreement with the changes introduced in the orientation kinematics. Efficient models for addressing these difficulties need for further developments.

The question concerning the rheological behaviour where the matrix exhibits elasticity is also an open problem.

\section{TOWARDS FORMING PROCESSES SIMULATION}

By assuming one of the models just described one could simulate several forming processes, as injection, extrusion or spin coating among many others. Any of the existing simulation techniques successfully applied for simulating processes involving short fibers suspensions could be used for evaluating the induced microstructure during the material forming. However, the comparison between the numerical predictions and experiments is a tricky problem because the characteristic dimensions of CNTs that makes practically impossible to have access to the microstructure.

In [11] we performed some preliminary simulations of spin coating by assuming treated CNTs and then the kinetic theory model involving the orientation distribution. These simulations were performed by using a meshless method in order to account for the large geometrical transformations and also the free and moving surfaces.

The use of CNTs in structural parts could be an appealing solution for enhancing many properties. Thus, by injecting a suspension of thermocuring resin and 
functionalized CNTs into a preform one could expect to perform a part with enhanced electrical properties for example. In general the injection of a resin into a preform is modelled as the flow of a viscous fluid in a porous medium. By combining the Darcy's law

$$
\mathbf{v}=-\mathbf{K} \times \nabla P
$$

and the fluid incompressibility

$$
\nabla \times \mathbf{v}=0
$$

it results

$$
\nabla \times(-\mathbf{K} \times \nabla P)=0
$$

that defined an elliptic partial differential equation that can be solved with appropriate boundary conditions for computing the pressure field, and then from Eq. (12) the velocity field that can be used in order to update the fluid domain.

Now, if we assume that the injected fluid is no more a simple viscous fluid but a CNTs suspension the just presented model is no more valid. Obviously, one could assume a pragmatic approach by measuring experimentally the equivalent permeability and then applying the previous model (Eqs. (12) and (14)). Even if there is no works concerning such an approach, one could expect that that simplified model could perform quite accurate predictions concerning the mould filling. In fact, the filling process kinematics is mainly controlled by the incompressibility condition, and then the rheology has a secondary role. Thus, we can expect quite good predictions concerning the evolution of the flow domain, bur poor predictions of the pressure field. However, the prediction of enhanced properties needs an accurate evaluation of the microstructure state, and in particular the tubes orientation distribution. Obviously, the model described by Eqs. (12) and (14) cannot describe such an evolution because the tubes orientation, and eventually its aggregation and/or disaggregation, are strongly affected by the microscopic flow kinematics. For example the model (12)-(14) predicts a null shear rate in the mould thickness with the consequent impact on the orientation mechanisms.

The use of at least a two scales simulation for coupling flow kinematics and microstructure evolution is at present an open problem, but without any doubt this topic will develop in the next few years.

\section{CONCLUSION}

The application of CNTs for enhancing properties of conformed parts or even to address innovative solutions to new and old ideas is growing. The optimisation and control of processes involving CNTs suspensions need accurate models describing the rheological behaviour of such suspensions. Some attempts in this directions has been proposed recently, but numerous questions remain open: explanation of linear viscoelasticity, CNT dispersion, flow and aggregation coupling, or the modelling of aggregation / disaggregation of non treated
CNTs. Concerning the forming process simulation, the standard simulation tools developed for simulating polymer melts of short fiber suspensions can be directly used as soon as the rheology of CNTs suspensions is assumed known. Thus, the injection, extrusion ... of CNTs suspensions can be accurately simulated using the models here described. However, other kind of applications, in particular those involving the flow of a resin into a perform, as encountered in usual composites forming processes, the existing models are not adequate for addressing the microstructure evolution, because the too simplified nature. Thus, the simulation of this kind of processes needs new multiscale approaches.

\section{REFERENCES}

[1] S. Iijima, Helical microtubules of graphitic carbon. Natute, 354, 56-58 (1991).

[2] Y.Y. Huang, S.V. Ahir, E.M. Terentjev, Dispersion rheology of carbon nanotubes in a polymer matrix. Phys. Rev. B 73, 125422:1-9 (2006).

[3] C.A. Dyke, J.M. Tour, Overcoming the insolubility of carbon nanotubes through high degrees of sidewall functionalization. Chem. Eur. J. 10, 812817 (2004).

[4] F. Folgar, Ch. Tucker, Orientation behaviour of fibers in concentrated suspensions, Journal of Reinforced Plastics and Composites, 3/2, 98-119 (1984).

[5] A. Ma, C. Cruz, A. Giner, M. Mackley, G. Regnier, F. Chinesta, Modeling elastic behavior in functionalized carbon nanotube suspensions, 11th. ESAFORM Conference on Material Forming (Lyon), 2008.

[6] A. Ma, F. Chinesta, M. Mackley, The rheology and modelling of chemically treated carbon nanotube suspensions, Journbal of Rheology, In press.

[7] A. Ma, M. Mackley, F. Chinesta, The microstructure and rheology of carbon nanotube suspensions, International Journal of Material Forming, 2, 75-81, (2008).

[8] A. Ma, F. Chinesta, A. Ammar, M. Mackley, Rheological modelling of carbon nanotube aggregate suspensions, Journal of Rheology, 52/6, 1311-1330, (2008).

[9] A. Montesi, D. Morse, M. Pasquali, Brownian dynamics algorithm for bead-rod semiflexible chain with anisotropic friction, The Journal of Chemical Physics, 122, 084903, (2005)

[10]C. Cruz, A. Ma, G. Regnier, F. Chinesta, M. Mackley, Comportement rhéologique des suspensions de nanotubes de carbone mono feuilletés fonctionnalisés, $43^{\text {ème }}$ Colloque du Groupe Français de Rhéologie, Ecole Polytechnique, Palaiseau, 2008.

[11] E. Cueto, A. Ma, F. Chinesta, M. Mackley, Numerical simulation of spin coating processes with functionalize carbon nanotube suspensions, International Journal of Material Forming, 2, 89-99, (2008). 